\title{
PRÁCTICAS EN LA ENSEÑANZA DE LAS CIENCIAS. PROBLEMAS Y POSIBILIDADES
}

\author{
MIGUENS, M. y GARRETT, R.M. \\ Universidad de Bristol, School of Education.
}

\section{SUMMARY}

We have effected an analysis of the role attached to practical studies in teaching sciences through the study of works published on this subjetc.

The work has been structured starting from the historic development, to go on with the ends and advantages and finishing it outstanding some of the aspects of what can be considered the core of practical work. Finally, we highlight the main objectives to establish these practical classes.

\section{INTRODUCCIÓN}

Las prácticas en la enseñanza de las ciencias han sido un asunto, casi siempre, sin controversia. Algunas veces, los profesores de ciencias hacen prácticas sin una buena razón o sin pensar en objetivos útiles. Con frecuencia toman esa opción en base a la creencia fuertemente mantenida de que las actividades prácticas son necesarias, importantes y características «sine qua non» de una buena enseñanza.

Una breve referencia at «estado de las artes» en lnglaterra y Portugal es el punto inicial que permite un más detallado análisis del problema, las contradicciones y debilidades de las actividades prácticas en la enseñanza de las ciencias a los alumnos de 11-13 años.

Un amplio entendimiento de los fines de las prácticas requiere una clarificación del significado de los varios tipos de actividades prácticas, que son diferentes en naturaleza y pueden ser empleadas en distintas formas y utilizadas para lograr diversas metas.

Una base fuerte de argumentación sería favorecer la necesidad de incrementar la utilización de proyectos, investigaciones o resolución de problemas de «fines abiertos» en la clase de ciencias para los alumnos de 11 13 años. Esta sugerencia no significa el abandono di otras actividades prácticas, significa que necesitamos valorar de nuevo el'Iugar que ocupan y la ventaja de los objetivos que pueden ser conseguidos a través de los diferentes métodos de las prácticas en ciencias.
1. TRABAJO PRÁCTICO EN LA ENSEÑAN. ZA ESCOLAR DE LAS CIENCIAS: UNA LARGA HISTORIA

Las expresiones «trabajo práctico», "actividades prácticas», «trabajo en laboratorio» o simplemente "prácticas», se utilizan aquí para indicar: el trabajo realizado por estudiantes en la clase o en actividades de campo, que pueden o no involucrar un cierto grado de interaccion del profesor, e incluye demostraciones, auténticos experimentos exploratorios, experiencias prácticas (experimentos normales en la escucla) e investigaciones (proyectos que encierran un número de actividades).

Profesores, elaboradores del currículo y autoridades educativas han mostrado una fe casi ciega en el trabajo práctico, con frecuencia sin una manifestación clara de su papel u objetivos (Reid y Hodson 1987). Tradicionalmente, al menos en Gran Bretaña y en los Estados Unidos, forma una gran parte de la enseñanza de las ciencias.

El valor del trabajo práctico fue amplificado hace más de cien años (Lock 1988), pero-como Kerr señala-talcs reclamaciones «nunca han sido cumplidas» (Kerr 1963, p. 9). Hasta el comienzo de esta centuria, las prácticas jugaron, en gran medida, un papel de apoyo, siendo empleadas para confirmar la teoría ya enseñada, y con la aparición del heurismo de Amstrong «el trabajo práctico central en la investigación y la genuina pregunta conduciría al entendimiento de la teoría» (Lock 1988). 
Entre las dos gucras mundiales, el trabajo práctico y su valide $z$ fue fuertemente cuestionado, pero la llegada de propuestas en la clase de ciencias del descubrimiento guiado a ambos lados del Atlántico, simbolizadas por proyectos como BSCS en los Estados Unidos y Nuffield en Gran Bretaña, estimuló otra vez la extensión del uso de actividades prácticas. En ese tiempo, la meta principal de alumnos descubriendo conceptos y principios, fueron buscados con el apoyo del maestro como una guía, una fuente, un medio de aprender al descubrir.

Recientemente, en 1985, el papel de las actividades prácticas en las lecciones de ciencias fue resaltado por ei Departamento de Educación y Ciencias en Gran Bretaña, cuando afirma que: «la característica esencial de educación en ciencias es que introduce a los alumnos al método científico» (DES 1985, p. 3) y «la educación en ciencias debería dar al mismo tiempo, a través del trabajo práctico u otro medio, oportuniđades para la adquisición de conocimientos y su comprensión» (DES 1985, p. 4).

In Portugal, se supone que el trabajo práctico hecho en las lecciones de ciencias no es suficiente, y en el lapso de 6-15 años un procedimiento de "manos-a-la-obra» se encuentra raramente en las escuelas.

Proyectos como Nuffield y BSCS tuvieron una fuerte influencia en los educadores de ciencias y en los programas para la educación de macstros en las escuelas de Portugal. Sin embargo, la escasez de recursos y facilidades, junto a la existencia de programas centralizados con tendencia a un contenido extenso y una tradicional y socialmente alta consideración didáctica de la enseñanza de las ciencias basada en Ios conocimientos, condujeron a los profesores a evitar las prácticas.

Tal situación parece contradecir las prescripciones generales del currículo y sugerencias metodologicas publicadas por las autoridades educativas. Por ejemplo, el actual programa de ciencias para 11-13 años afirma: «los temas tratados en cada lección no deberán ser presentados como un cuerpo de conocimiento ya fijado, dirigiendo a los alumnos a actitudes pasivas» (MFC 1981, p. 30).

Más aún, el mismo documento sugiere «el uso del método experimental» con el fin de lograr un ambiente de aprendizaje que estimule una actitud investigadora, la intervención de habilidades manipulativas c intelectuales y la promoción del pensamiento crítico.

Aun así, un estudio reciente llevadio a cabo por el gabinete de Isstudios y Planificación (GEP) ha revelado que solamente 17 profesores de ciencias (de 48 entrevistados) usan trabajos prácticos durante más del $20 \%$ del tiempo, mientras 8 profesores no hicieron ninguna práctica(Valente 1989). Todos los profesores utitizaron menos del $17 \%$ del tiempo para demostraciones y la mayoría empleó menos del $10 \%$ de su tiempo en organizar trabajo práctico. No sorprende que la mayoría de los profesores de ciencias utilizaran del 20 al $50 \%$ de su tiempo disponible en explicación verbal (Valente 1989).

Al contrario de la situación portuguesa, en Inglaterra y Gales, el $83 \%$ de escuelas gastan del 40 al $80 \%$ de su tiempo en trabajo práctico (Beatty and Woolnough 1982), y no más del $5 \%$ de los profesores de ciencias cuestionaron seriamente el valor de trabajo práctico individual (Kerr 1963). Aún más, en sus comentarios, los profesores apoyan fuertemente esta manera de enseñar:

«Sin trabajo práctico, física no es física»o «Una vez visto, nunca olvidado" son comentarios típicos del estudio de Kerr en los años 60.

\section{TRABAJO PRACTICO: FINES Y VENTAJAS}

No es suficiente decir que los profesores utilicen más o menos actividades prácticas en las lecciones de ciencias. Preguntas importantes como: ¿Por qué los profesores utilizan trabajo práctico? o ¿Qué tipo de prácticas ven ellos válidas?, deben ser tratadas.

Comparando los trabajos de Kerr, Beatty y Woolnough, puede observarse que los profesores seleccionan diferentes objetivos y tratamientos para su trabajo práctico (Kerr 1963, Beatty y Woolnough 1982).

Beatty y Woolnough (1982) presentaron a los profesores una lista de veinte objetivos seleccionados de trabajos previos de Kerr (1963) y de Thompson (1975), y encontraron que los profesores, trabajando con los alumnos de 11-13 años, estimaron mucho más alto aquellos objetivos relacionados con destrezas prácticas seguidos por aptitad para la ciencia. Pero una diferencia significativa entre los hallazgos originales de Kerr y los del equipo de Oxford es que los objetivos de las prácticas relacionados con la teoría, o como soporte de ella, han perdido significancia y son ahora considerados menos importantes.

Mientras Kerr loclasificó como una «evidencia en apoyo de la necesidad para una integración íntima de trabajo práctico y teorético» (Kerr 1963,p. 31), Beatty y Woolnough, casi veinte años después, hallaron que «el trabajo práctico es considerado por los profesores más como una actividad relacionada con el desarrollo de habilidades que por st naturaleza son específicamente prácticas, y menos como un método de ampliar el conocimiento teórico o de comprenderlo» (Beatty y Woolnough 1982).

De hecho, considerando los objetivos que resaltan el trabajo práctico como una ayuda a la teoría en comparación con los relacionados con el desarrollo de destrezas prácticas, se cncontró que mientras todos los del primer grupo han disminuido de valoración, todos los del segundo grupo han aumentado (Beatty y Woolnough 1982). Sin embargo, ellos sugieren que no hay cvidencia do un cambio dramático en las razones dadas por los profesores para hacer trabajo práctico a pesar de todas las innovaciones curriculares que han tenido lugar entre estos dos estudios. Una más completa discusión de las razones dadas por los profesores para hacer trabajo práctico en diferentes niveles puede encontrarse en Bound ot al. (1980), Buckley y Kempa (1971), Gould (1978), Thompson (1975), West (1972) y Woolnough (1976).

La segunda cuestión a considerar aquí es aquélla de los diferentes procedimientos para prácticas en las clases de 
ciencias escolares. Los profesores pueden elegir demostraciones, experimentos de descubrimiento (más o menos guiados), experiencias prácticas e investigaciones o proyectos. Frecuentemente estas diferentes formas de actividades prácticas entran en la denominación de trabajo práctico, pero las implicaciones en la enseñanza y aprendizaje para cada uno de estos procedimientos son bastante diferentes y los objetivos alcanzados con cllos deben ser claramente definidos. Volveremos a este punto más adelante.

Primero, consideremos lo que sucede en Inglaterra, usando una vez más los estudios de Kerr y Beatty. Kerr encontró que el trabajo práctico en el primer y segundo años consistía predominantemente en demostraciones, pero este tipo de actividad fue abandonada en los años superiores (Kerr 1963, p. 54). Además, actividades de verificación fueron hechas frecuentemente, aunque los profesores hicieron referencia a su valor educativo. Investigaciones se hicieron raramente, aunque los profesores les asignaron de un alto valor educativo.

Por otro lado, Beatty y Woolnough (1982) encontraron que las escuelas utilizaban ejercicios estándar más cue experimentos de descubrimiento y ambos más que demostraciones. Tal tendencia parece indicar que las demostraciones, no sorprendentemente, fueron sustituidas por mayor motivación, «manos-a-la-obra» y procesos activos de realizar trabajos prácticos.

Sin embargo, proyectos de trabajo, investigaciones o resolución de problemas con fin inconciuso no fueron comúnmente utilizados en las escuelas investigadas por el equipo de Oxford. De hecho, el $79 \%$ de las escuelas gastaron menos de cinco horas al año en proyectos de trabajo. Aun así, esto representa un aumento significativo desde 1962 (Beatty y Woolnough 1982). Resultados similares se encontraron en escuelas escocesas (Wilson 1977).

\section{PROBLEMAS CON LAS PRÁCTICAS}

Las situaciones descritas en el primer apartado muestran lo que puede ser considerado como dos extremos repre:sentados por escuelas inglesas y portuguesas, y puede ser que una posición intermedia, en alguna parte entre $\mathrm{GI}$ uso intenso đe prácticas «manos-a-la-obra» en Gran Bretaña y la aparente baja utilización de taies actividades en Portugal, sea más realística. Por cierto, la Asociación Británica para liducación en la Ciencia ha sugerido que:

«En años recientes la significancia de "haciendo" ciencia ha conducido al abandono de clases clásicas de demostración, y la sobrevaloración de las clases prácti. cas ha forzado a los profesores y alumnos a cuestionar la validez de las sesiones de discusión o los estudios de: ciencia basados en la bibliograffa» (ASE 1981, p. 29).

Sin embargo, mientras se favorece una reconsideración de los procedimientos alternativos a la enseñanza de las ciencias sin incluir trabajo práctico, la ASE, en su documento «Educación a través de la Ciencia» cuidadosamente declara que esto no «implica ninguna devaluación de la importancia de las sesiones de laboratorio bien organizadas que han sido bien planificadas para lograr objetivos que solamente se alcanzan a través de la utilización de un procedimiento de "manos-a-Ia-obra"." (ASE 1981, p. 29).

Este uso o impensado abuso de prácticas y demandas al llamado procedimiento de procesos ha sido ampliamente cuestionado durante un cierto tiempo en Europa, USA, Nueva Zelanda y Australia, y ha conducido a Ia identificación de una varicdad de problemas asociados con el trabajo práctico que puede ser visto descie perspectivas filosóficas, psicológicas y pedagógicas.

\section{Problemas con la ciencia}

El tratamiento más ingenuo de las prácticas supone que el conocimiento científico no es problemático y expuesto al descubrimiento a través de sencillas observaciones imparciales y de la correcta aplicación del método científico (Hodson y Reid 1988). El procedimiento de descubrimiento parece caer en la trampa inductivista de considerar la observación como objetivo y como el punto de partida del método científico.

Sin embargo, tal punto de vista puramente inductivista es fuertemente refutado por la filosofía actual de la ciencia. Todas están de acuerdo en que la observación está cargada de teoria y que no hay un método científico unificado.

Además, si tal método existiera, no siempre comenzaría con observacioncs. Los científicos no se mueven nece* sariamente de lo concreto a lo abstracto o siguen pasos o procesos secuenciales, como se supone en la mayoría de las prácticas de ciencias en la escuela.

Hodson (1988) al discutir el papel y posición de la observación científica arguye por un enfoque de nuestra atención a: 1) el reconocimiento de que la observación no es fide-digna y depende de la teoría, 2) el hecho de que las técnicas de la observación científica tienen que ser aprendidas, 3) la necesidad de tener en cuenta la existencia de estrategias de los niños, 4) la reconsideración de la idoneidad del procedimiento por descubrimiento, y 5) un rechazo de la imagen de la ciencia como estudio libre de valores.

Driver pone las mismas ideas en una pregunta: « ¿Cómo puede un observador inexperto distinguir lo que llamamos observación significativa de lo que es accidental, cuando lo significativo viene de la interpretación teórica que nosotros impondremos sobre los sucesos?" (Driver 1975).

Así, como Wellington (1981) ha señalado, aun exponiendo a nuestros alumnos a una gran cantidad de datos a través de cuidadosas observaciones, en diferentes condiciones y situaciones, parece ingenuo esperar que ellos obtengan conceptos científicos abstractos. Ios conceptos no pueden ser obtenidos o descubiertos a través de experiencias, aunque puedan ser aplicados útilmente a ellas. Científicamente, no puede generali- 
zarse a partir de resultados científicos. Normalmente la teoría los predice y entonces se trabaja sobre sllos.

Si el trabajo práctico utiliza el tratamiento inductivista, estamos sugiriendo con frecucncia a nuestros alumnos «el juego de la respuesta correcta» (Driver 1983), que ellos rápidamente aprenden a jugar, con resultados no satisfactorios para la enseñanza de la ciencia o del aprendizaje.

\section{Problemas con el aprendixaje}

Estudiando las actividades de la enseñanza de las ciencias y sus problemas específicos, Tasker (1981) identificó una serie de ellos que pueden resumirse en:

Los alumnos consideran las lecciones como hechos aislados mientras los profesores las relacionan como una serie de experiencias.

I os alumnos perciben objetivos de la tarea, diferentes de los percibidos por el profesor.

Las percepciones de los alumnos sobre la naturaleza del trabajo no incluye características de crítica científica, pero los profesores suponen que ellos la hacen.

Ios ahmnos carecen del conocimiento previamente supuesto y no son capaces de alcanzar el «estado mental» requerido.

Los resultados logrados por las percepciones de los alumnos relativas a lo significativo del trabajo no son aquéllos que los profesores suponen que son percibidos (Tasker 1981).

Fsta lista puede condensarse, quizás de una forma muy simpijificada, en considerar los puntos de vista de los alumnos, las percepciones y preconceptos llevados a la clase de ciencias que parecen afectar no solamente al aprendizaje de los conceptos de ciencias, sino a la forma de hacer las actividades prácticas de ciencias.

Así, si el aprendizaje se ve como un proceso activo en el que las creencias previas, ideas y conceptos, juegan un importante papel, Ios alumnos utilizan sus conocimientos previos cuando observan, diseñan y realizan experimentos e interpretan resultados.

Como Millar y Driver sugieren: «Parece que lo que los alumnos observan, lo que hacen y las interpretaciones que ellos dan, dependen de los conceptos que ellos utilizan» (Millar y Driver 1987).

Así, los alumnos necesitan unir las prácticas a su estructura conceptual, si no, las recordarán como una serie de experiencias desconectadas (Driver 1983).

El trabajo de Assessment Performance Unit UK da fuerza a tales demandas identificando dos formas en que Ios puntos de vista del mundo de los alumnos puede por el contrario afectar a su realización:

«Primeramente si la situación presentada no encaja en un esquema aceptado, entonces lo que se observa como relevante se desconoce. En segundo lugar, si la situación se observa de acuerdo con su experiencia previa del mundo y los aluminos sienten confianza en su experiencia, frecuentemente, responderán dando información que no es obscrvabie.» (APU 1984, p. 31).

\section{Problemas pedagógicos}

Los problemas pedagógicos con los trabajos prácticos están principalmente relacionados con la enseñanza de habilidades cientifficas que se utilizan con frecuencia, como un argumento para hacer actividades prácticas.

Un problema central surge por las dudas sobre la posibilidad de transferir esas habilidades.

Wellington, por ejemplo, señala que destrezas como pensar, razonar, resolver problemas, observar e inferir, se supone frecuentementc que son transferibles o separables del conocimiento, experiencias, actitudes, conceptos y teorías a las que ellos están ligados. Este «atomizado» tratamiento no parece estar en concordancia con el necesario punto de vista holístico de «haciendo ciencia». Tal posición está ampliamente desarrollada por Woolnough quien argumenta para un gestalt práctico, recordándonos que el todo es, frecuentemente, más, y diferente que la simple suma de partes (Woolnough 1989).

\section{Problemas con evidencia investigada}

En esta etapa es natural preguntarse qué dice la investigación sobre la efectividad de «manos-a-1a-obrat», méto. dos activos, trabajo de laboratorio o cualquier otro nombre que se ponga a las actividades prácticas de ciencia.

Bredderman considera que existen pruebas consistentes para sugerir que:

«Más tratamientos de actividades basadas en procesos en la enseñanza de ciencias ganan a los métodos tradicionales en que un amplio número de estudiantes consiguen otros niveles.» (Bredderman 1983).

En una breve recopilación de la literatura sobre el papel del trabajo de laboratorio, Blosser señala algunos resuitados, tales como:

Las actividades de laboratorio parecen ayudar a los estudiantes que cstán en un nivel medio o bajo.

La instrucción del laboratorio incrementó la habilidad de los estudiantes para resolver problemas.

El nivel de éxito en ciencias era superior para los estudiantes de 10 años que habían observado y realizado experimentos.

Las actividades de laboratorio pueden desarrollarse para crear una situación que estimule el desarrollo cognitivo (Blosser 1983).

En un estudio comparativo de actividades hacia la ciencia entre profesores y estudiantes de nivel elemental en 
clases basadas en procesos y en clases tradicionales, Kyle et al., encontraron que mientras los dos grupos de profesores tenían similar percepción de la ciencia, fuertes diferencias actitudinales se encontraron entre los estudiantes (Kyle et al. 1988).

Finalmente, en un reciente estudio de los efectos de los métodos "manos-a-la-obra» y las demostraciones de laboratorio del profesor, en relación a la habilidad de razonamiento y conocimiento previo, Glasson ha encontrado que los dos métodos resuitaban iguales en conocimientos (el dominio de los hechos y conceptos de una materia), pero los estudiantes de clases de laboratorio en «manos-a-la-obra» reajizaban significativamente mejor los tests de procedimientos de conocimiento (razonamiento de estrategias aplicadas a la resolución de problemas en orden a generar soluciones para los problemas) que los estudiantes de clases de demostraciones del profesor (Glasson 1989).

Se puede decir que ésta es una relación muy positiva de conclusiones de investigaciones sobre la efectividad del trabajo práctico como método de enseñanza. Sin embargo, posiciones más escépticas han sido adoptadas por Garrett $y$ Roberts (1984), quienes hallaron que un pequeño grupo de trabajo práctico sobre el total no era más eficaz en los conceptos de enseñanza de las cicncias que lo eran las clases de demostración. Más recientemente, Hodson (1990), citando a Holstein y Lunetta (1982), argumenta que «la mayoría de los estudios sobre cficacia del trabajo práctico han sido defectuosos por pobre diseño experimental, inadecuado control de variables y el uso de tests inapropiados». Así, él concluye que hay argumentos teóricos consistentes y pruebas de investigación para reforzar el punto de vista de que «el trabajo práctico en la enseñanza de ciencias, cómo está organizado actualmente, es ampliamente improductivo y patentemente incapaz de justificar las frecuentes exageradas denandas efectuadas para él» (Hodson 1990).

\section{TRABAJO PRÁCTICO EN LA ENSEÑAN. ZA DE CIENCIAS: QUE CAMINO AHORA?}

¿Significa la existencia de estos problemas que las actividades prácticas deban ser abandonadas?

No se pide una acción drástica, pero necesitamos volver a pensar el papel, naturaleza y objetivos de las prácticas en nuestras clases. Como se ha dicho anteriormente, el trabajo práctico puede ser interpretado en una amplia extensión de formas. Los profesores pueden tratar las prácticas, aun el mismo experimento, de una variedad de formas y de acuerdo con los objetivos que ellos tengan en mente. El objetivo central del trabajo práctico se encuentra dentro del tratamiento y el contexto con que los profesores usen Ia amplia variedad de actividates prácticas que estén disponibles.

De hecho, el trabajo práctico en la mayoría de los casos está limitado a ejercicios en los que los estudiantes realizan alguna clase de actividad o manipulación de equipos guiados por claras y precisas instrucciones. Este trabajo, frecuentemente, conduce a los alumnos a un seguimiento mecánico de pasos, en fichas o carpetas de trabajo, sin que ningún pensamiento sea aplicado $\mathrm{y}$, consecuentemente, lo que se comprende es mínimo. Esta especie de ejercicios de «cocina», con estudiantes siguiendo recetas, puede tener alguna limitada utilidad de enseñanza de la ciencia y aprendizaje, pero se debe tener claro el cscaso valor que representan y que a menudo los objetivos que ilustran o verifican pueden ser conseguidos por otros medios. Cuando estas actividades para prácticas de observación, medida o manipulación en relación a desarroltar destrezas prácticas básicas y a involucrar a los estudiantes en las técnicas diversas que los científicos utilizan en su trabajo, no se deberían enmascarar como experimentos (Woolnough y Alsop 1985).

Experimentos de descubrimiento guiado, en que los procedimientos son realizados por los estudiantes a una predeterminada y simple respuesta correcta, tampoco son mejores. I a mayoría de los probiemas discutidos anteriormente son característicos de actividades de descubrimiento guiado. Leyes científicas, principios o conceptos no son, generalmente, «descubiertos» ni «redescubiertos" por los alumnos y la característica convergente de esos experimentos conduce al «juego de la respuesta correcta» ya mencionado. Este tipo de trabajo práctico, a menudo asociado con algunos acercamientos ingenuos a la ciencia NUFFIELD, no parece de mucha ayuda en la enseñanza y aprendizaje de la ciencia y lieva la marca indeleble de los puntos de vista inductivista y empirista de la ciencia, que no está de acuerdo con la filosofía actual de la ciencia. Además, pueden conducir a la frustación de los alumnos cuando, sistemáticamente, fallan para conseguir «la respuesta correcta». O por el contrario, ellos encuentran rấpidamente que están descubriendo la certeza, entran en el juego y lo hacen de acuerdo con las reglas de profesor.

Demostraciones, realizadas por el profesor a un grupo de estudiantes, involucrando o no alguna discusión sobre lo que está haciendo, han sido casi abandonadas. Una posible razón para tal hecho es la asociación de demostraciones con métodos didácticos y de exposición. Sin embargo, este tipo de prácticas puede ser útil en una amplia extensión de situaciones.

Johnston and Wham (1980) consideran que la demostración es «fuerte en su capacidad para ilustrar la teoría». Connell (1971) se refiere a un gencral acuerdo sobre la existencia de circunstancias en que la demostración es un apropiado tratamiento. Woolnough y Allsop, aunque no consideran ta demostración uno de sus tres principales tratamientos de las prácticas, admite que puedan ser útiles en: «ayudar a los estudiantes a hacer las reuniones fundamentales entre realidad y teorías abstractas, y capacitar al alumno a construir un marco cognitivo más estructurado e interconectado» (Woolnough y Alisop 1985, p. 63).

En una revisión de la literatura, Gartett y Roberts (1982) conclúan que la demostración es algunas veces necesaria y deseabte, particularmente, cuando se consideran materiales de elevado costo, procedimientos peligrosos y el 
propio manejo del equipo.

Las experiencias definidas por Woolnough (1983) como simples experimentos, exploratorios, generalmente cuaJitativos, muy cortos y rápidos, pueden ser interesantes y actividades beneficiosas en relación a dar a los alumnos un primer acercamiento al fenómeno científico.

Como Woolnough y Alısop scr̃alan: «Unos pocos minutos experimentando un fenómeno determinado, permitiendo a la vez pensar y discutir sobre él, es frecuentemente tiempo (...) bien empleado (Woolnough y Alsop 1985, p. 56). Así, con esas simples experiencias y a condición que haya oportunidades para discutir y asimilar, los estudiantes obtienen un acercamiento al fenomeno que conducirá a la comprensión y creencia.) (Woolnough y Allsop 1985).

Trabajo de campo, en el que los estudiantes salen del laboratorio, de la escuela, y trabajan explorando, recogiendo materiales y datos, experimentando en cl campo, como un ecologista o un geólogo lo harían (1 ock 1987), debe ser también considerado.

Desde que los fines del medio ambiente tienen un lugar significativo en los currículos de ciencias, deberían ser explorados por trabajos de campo tanto en el ambiente urbano como en el rural. T'ales estudios pueden ser utilizados como puntos de arrancue para discutir algunos de los fines contradictorios en ciencia, en política, económicos, tecnológicos y sociales, y la importancia de la ciencia en el medio ambiente.

Además, en un currículo de ciencias que tiene un componente significativo de ciencias de la tierra, hay muchos temas que sería bencficioso explorar fuera del laboratorio, particularmente, por ejemplo, en los sistemas de España y Portugal.

Las investigaciones en que los estudiantes están involucrados en resolver nuevos problemas, buscando, investigando, estudiando con más o menos profundidad los temas relacionados a un problema particular y encontrando posible soluciones.

Realizando tales investigaciones, proyectos o actividades de resolución de problemas abiertos-cerrados, tos alumnos están trabajando y resolviendo problemas como científicos.

Aquí reside, nosotros argumentamos, el mérito real, el verdadero valor, la utilidad y el significado del trabajo práctico.

Esos estudios experimentales verdaderos requieren que los alumnos se adueñen de los problemas a estudiar y que Ios reconozcan como problemas reales. Entonces, los alumnos se involucran en planificar, ejecutar, interpretar y evaluar las pruebas o las posibles soluciones, y exponer sus resultados, tanto de forma escrita como verbal (Lock 1987).

Fste accrcamiento divergente a las prácticas evita juegos de «respuesta correcta» o «descubrimiento de certeza» y puede ser llevado a cabo por los estudiantes individual- mente o en pequeños grupos, en un período corto o largo, y unido o no al contenido que se está cstudiando.

Como Hodson y Reid dicen: «Aquí cl énfasis no está sobre el aprendizaje de los métodos de ciencia o en desarrollar destrezas en los procesos individuales de la ciencia, sino en usar los métodos y procesos de la ciencia para investigar fenómenos, resolver problemas y continuar el interés de lo que los alumnos han elegido.» (Hodson y Reid 1988).

Esta implicación de los alumnos en llevar a cabo sus propias investigaciones no busca establecer, ilustrar o verificar un principio o ley cientifica, sino darles ta oportunidad y la experiencia de planificar un experimento, utilizar su propia iniciativa, enfocar el diseño del experimento, elegir y usar los recursos, recoger cuidadosamente los datos, e interpretar los resultados (Driver 1983, p. 81 ).

Los estudiantes encuentran los proyectos altamente motivadores, interesantes, estimulantes, divertidos y relevantes (Swain 1977) y el interesante trabajo de investigación que se está llevando a cabo en Inglaterra en $11-13$ años (Woolnough y Allsop 1985), como resultado de su involucramiento y dedicación entusiastica, muestra como las investigaciones pueden ser de valor y factibles.

$Y$, una vezmás, si aceptarnos un acercamientoconstructivista a la ensenanza y aprendizaje de la ciencia, las investigaciones, proyectos o actividades de resolución de problemas abiertos-ccrrados, los estudiantes los sentirán como pro blemas realcs, involucrándose en una investigación personal, conducida en gran parte por su propia iniciativa, dándoles justamente una gran parte de la responsabilidad en las actividades de su aprendizaje. Esto es totalmente compatible con el necesario compromiso activo del que aprende en construir y generar explicaciones sobre el mundo.

Aún más, como señala Lock: "La introducción de investigaciones y trabajo de campo en cl curriculo de ciencias pucde ayudar a aumentar la confianza de profesores y alumnos para operar en situaciones donde las respuestas no están disponibles (...) y proporcionar un medio en que los fines locales y nacionales, sociales y medio ambientales puedan ser introducidos en el currículo de ciencias para 11-13 años." (Lock 1987, p. 66).

Es también a través de investigaciones y proyectos cómo el acercamiento holístico en la actividad científica sugerido por Woolnough (1989) puede ser alcanzado en ciencia escolar.

Tales actividades investigadoras, cuidadosamente preparadas por profesores y estudiantes, dan tiempo para la discusión y ponen atención efectiva a las fases del preexperimento y postexperimento (Driver 1983, Reid y Hodson 1987). Pueden también considerarse como una respuesta a las demandas de variedad en los trabajos prácticos (Johnston y Wham 1980).

Parece que la verdadera investigación en la ciencia escolar sería el mejor camino para liberar las actividades prácticas de la tiranía de la teoría (Woolnough 1983). De 
hccho, el interés de la unión entre enseñanza conceptual, teoría y actividades prácticas es al menos dudoso (Kreitler y Kreitler 1974, Donnelly 1979), y la opción para procedimientos investigadores puede facilitar el alejar las prácticas de la teoría.

No se sugiere que los profesores deberían usar las investigaciones para lograr varios objetivos diferentes simultáneamente, en realidad tanta falta de claridad en los propósitos no se debe considerar como una buena práctica (Reid y Hodson 1987). Debe señalarse, sin embargo, que una amplia variedad de propósitos puede lograrse con la realización de investigaciones de los alumnos en una clase de ciencias. Involucrando a los alumnos en trabajos prácticos, principalmente investigaciones, y ocasionalmentc actividades complementarias, ejercicios, experimentos exploratorios y demostraciones pueden alcan* zarse diversos fines. Una revisión de la literatura reciente sugicre que los objetivos de más valor para el tribajo práctico son los siguientes:

1. Desarrollarcompetencias en el trabajo como un científico real resuelve problemas. Desarrollar la habilidad para realizar una investigación científica genuina (Woolnough y Allsop 1985).

\section{REFERENCIAS BIBLIOGRÁFICAS}

APU, 1984. Science at Age 13 - Science report for tcachers: 3 London: DES.

ASE, 1981. Eduation through Science. Herts: ASE.

BEATTY, J. W. y WOOLNOUGH, B. E., 1982. Practical work in 11-13 Science: the context, type and aims of current practice, British Educational Research Journal, 8(1), pp. 23-30.

BOUND, D. J.,1DUNN, J., KENNEDY, T. y THORLEY, R., 1780. The Aims of Science Laboratory Courses a Survey of Students, Graduates and Practicising Scientists, European Journal of Science Education, 2, pp. 415-428.

BRFIDDERMAN, T., 1983. Effects of Activity-based Elementary Science on Student Outcomes: A quantitative Synthesis, Review of Educational Research, 53, 4, pp. 499-518.

BROOK, A., DRIVER, R. y JOHNSTON, K., 1989. Learning processes in Science: a classroom perspective, en Wellington, J. (cd.), Skills and Processes in Science Education. (Routledge: London).
2. Ayudar a los estudiantes a extender un conocimiento sobre fenómenos naturales a través de nuevas experiencias (Driver et al. 1985).

3. Facilitar a los estudiantes una primera experiencia, un contacto con Ia naturaleza y con el fenómeno que ellos estudian (Woolnough y Allsop 1985).

4. Dar oportunidades para explorar la extensión y límite de determinados modelos y teorías. Comprobar ideas alternativas experimentalmente y aumentar la confianza al aplicarlas en la práctica (Brook et al. 1989). Explorar y comprobar las estructuras teoréticas a través de la experimentación (Reid y Hodson 1987).

5. Desarrollar algunas destrezas científicas prácticas, tales como observar y manipular.

Obviamente, parece que las investigaciones aumentan los fines fundamentales del trabajo práctico. La mayor utilización de actividades de investigación y resolución de problemas debería ser la característica fundamental de las prácticas en la clase de ciencias, mientras otras formas de trabajos prácticos deberían tener un papel complementario y secundario en las actividades de cnseñanza de la ciencia.

BROSSER, P.E., 1983. The Rôle of the Laboratory in Science Teaching, School Science and Maths, 83 (2), pp. 165-169.

BUCKLFY, I. G. y KFMPA, R. F. 1971. Practical Work in sixth form chemistry courses - an enquiry, Science School Review, 52,182 , pp. 24-36.

CONNELL, L., 1971. Demonstration and individual practical work in science teaching: a review of opinions, Science School Review, 52, 180, pp. 692-703.

DES, 1985. Science 5-16; A Statement of Policy. (DES: London).

DONNELLY, J., 1979. The work of Poper and Kuhn on the nature of science, Science School Review, 212,60, pp. 489500 .

DRIVER, R., 1975. The name of the game, Science School Review, 56, pp. 800-805.

DRIVER, R., 1983. The Pupil as Scientist? (Milton Keynes: OUP).

GARREIT, R. M. y ROBERTS, I.F., 1982. Demonstration Vs. 
small group practical work in Science Education: a critical review of studies since 1900 , Studies in Science Education., 9, pp. $109-146$.

GLASSON, G. E. 1989. The Effects of Hands-on and Teacher Demonstration Laboratory methods on Science Achievement in Relation to Reasoning ability and prior Knowledge, Jownal of Research in Science Teaching, 26(2), pp. 121-131.

GOULD, C. D., 1978. Practical work in sixth form biology, Journal of Biological Education., I2(1), pp. 33-38.

HODSON, D., 1986. The nature of scientific observation, Science School Review, scpt., pp. 17-29.

HOISSON, D., 1990. A critical look at practical work in school science, Science School Review, 70, 256, pp. 33-40.

HODSON, D. y REH), D., 1988. Changing priorities in Science Futucation, Part II, Science School Review, 70, 25 h, pp. 159165.

JOHNSTON, A. y WHAM. A., 1980. A case for variety in practical work, Science School Review, 61, 217, pp. 762764.

KIER, I. F., 1963. Practical Work in School Science. (Leicester Univ. Press.: I eicester).

KREITI.FR, H. y KRFITLER, S., 1974. The rôlc of the experiment in Science Education, Instructional Science, 3, pp. 75-88.

KYLE, W. C., BOUNSTETILR, R. J. y GADSDEN, T., 1988. An implementation study: An analysis of elementary student's and teachers' attitudes toward science in process - approach Vs. Traditional Science classes, Jounal of Research in Sciche Teaching, 25, 2, pp, 103-120.

LOCK, R., 1987. Practical Work, en Foster, D. y Lock, R. (ed.), Teuching Science, 11-13. (Croom Helm: London).

LOCK, R., 1988. A history of practical work in school science and its assessment, 1860-1986, Science School Review, 70, 250, pp. 115-119.

MEC, 1981. Ensino Preparatbrio - Programas para o año lectivo 1981/82. (Direç̧åo Geral do Ensino Básico: Lisboa).

MILLAR, R. y Driver, R., 1987. Beyound Processes, Studies in Science Education, 14, pp. 33-62.

REID, D. y HODSON, D., 1987. Science for all. (Cassell: Lisboa).

SWAIN, J. R., 1977. Project work in Nufficld physical science, Science School Review, 58, pp. 570.578.

TASKER, $R$, 1981. Children's views and classroom Expericnces, Australian Science Teachers Journal, 27 (3), pp. 33-37.

THOMPSON, J.J. (ed.), 1975. Practical work in Sixthorm Science. (Science centre, Department of Education Studies: Oxford).

VALENTE, O., 1989. Práctica Pedagógica: análise da situaçâo. (GEP: Lisboa).

WELLINGTON, J. J., 1981. What's supposen to happen, sir?: some problems with discovery learning, Science School Review, 63,222, pp. $167-173$.

WELLINGTON, J. J. (ed.), 1989. Skills and Processes in Science Fducation: A critical analysis. (Routledge: Londres).

WEST, R. W., 1972, Objectives for practical work in school chemistry, Science School Review, 58, pp. 148-160.

WILSON, J. M., 1977. Practical work in Physics in Scotish schools, Science School Review, 58, 205, pp. 738-789.

WOOLNOUGH, B. E., 1976. Practical work in sixth form physics, Physics Education, 11, pp. 392-397.

WOOLNOUGH, B. E., 1983. Exercises, investigations and experiences, Physics Education, 18, pp. 60-63.

WOOLNOUGH, B. E., 1989. Towards a holistic view of processes in Science Education, in Wellington (ed.), Skills and Processes in Science Education: a critical analysis. (Routledge: London).

WOOLNOUGH, B. E. y AI,I.SOP, T., 1985. Practical work in science. (CUP: Cambridge). 